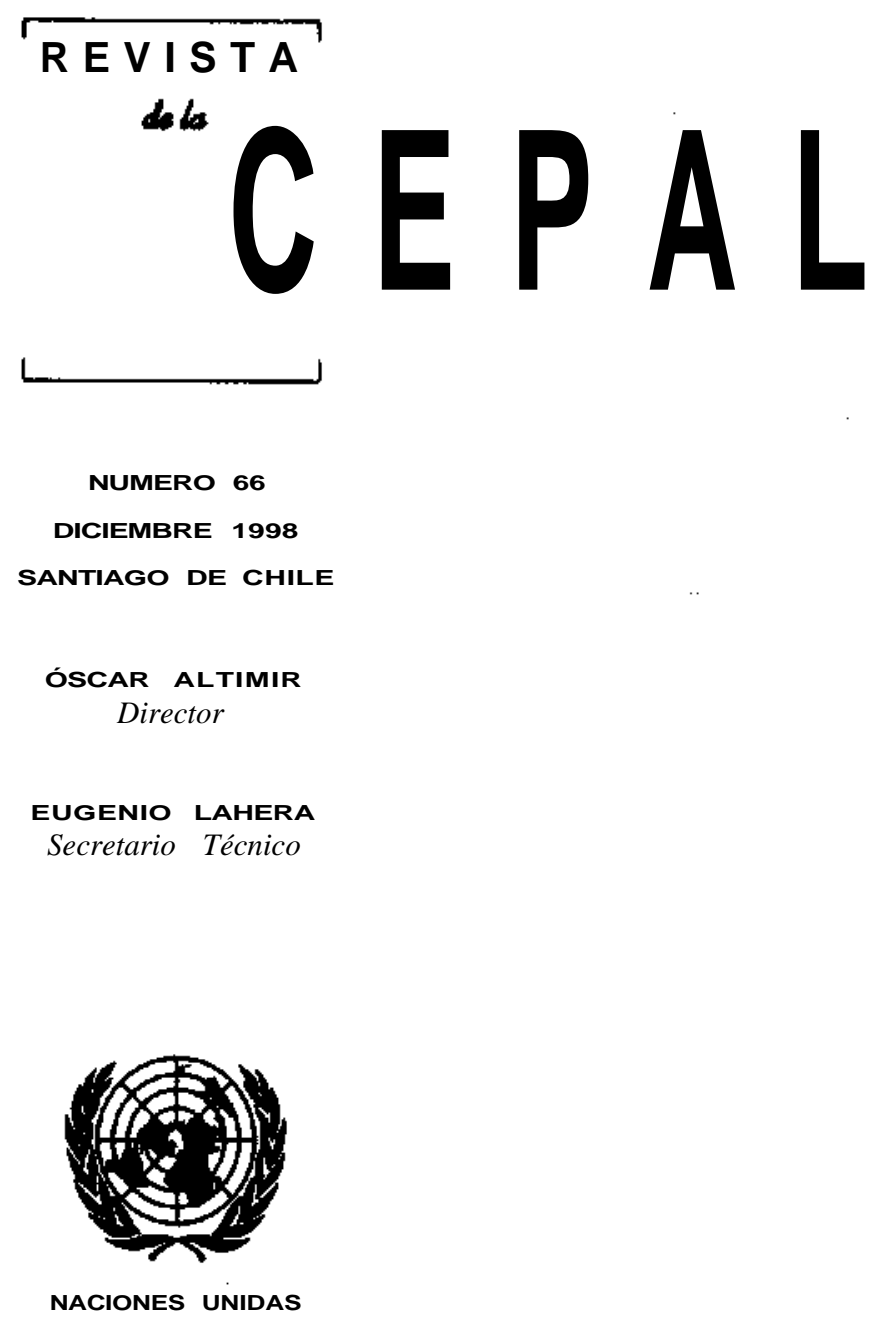


Más allá del Consenso de Washington: una visión desde la CEPAL

José Antonio Ocampo

La economía de Cuba

David ¡barra y Jorge Máttar

La educación en América Latina: la demanda y la distribución importan

Nancy Birdsall, Juan Luis Londoño y Lesley O'Connell

Determinantes de la desigualdad entre los hogares urbanos

Luis Felipe Jiménez L, y Nora Ruedi A.

Los compromisos de gestión en salud de Costa Rica con una perspectiva comparativa

Ana Sojo

Una estrategia de desarrollo a partir de complejos productivos en torno a los recursos naturales

Joseph Ramos

Grandes empresas y grupos industriales latinoamericanos

Celso Garrido y Wilson Peres

Entre el control político y la eficiencia: evolución de los derechos de propiedad agraria en México

Gustavo Gordillo, Alain de Janvry y Elizabeth Sadoulet

Los aranceles y el Plan Real de Brasil

Renato Baumann, Josefina Rivero y Yohana Zavattiero

Publicaciones recientes de la CEPAL 


\section{La educación en América Latina: la demanda y la distribución importan}

Nancy Birdsall

Asociada Principal,

Camegie Endowmentfor

International Peace

Juan Luis Londoño

Presidente, Revista Dinero

(Colombia)

Lesley O'Connell

Consultora, Banco

Interamericano de Desarrollo
Aunque los gobiernos de la región han aumentado sus gastos en salud y educación, los resultados son insatisfactorios. El gasto en esos servicios se ha considerado habitualmente corno transferencia y no como inversión. La acumulación de capital humano ha sido relativamente lenta, con efectos negativos sobre el crecimiento económico y se ha distribuido en forma dispareja entre los diversos grupos de ingreso, lo que ha aumentado la desigualdad. En este artículo se examinan las causas de estos resultados. En primer lugar se describe la naturaleza del problema, aportando datos que confirman la escasa e inequitativa acumulación de capital social en la región, la que ha acentuado la desigualdad en la distribución de la riqueza y los ingresos. Luego se discuten las causas que explican esos resultados y se pone de relieve el efecto de una débil demanda de educación entre los pobres, causada, entre otros factores, por las restricciones de su ingreso y la política antigua de protección económica. En las conclusiones se destaca que !as reformas económicas han aumentado la demanda de capital social lo que eleva la rentabilidad privada de la inversión en capital humano y da origen a un renovado interés del sector privado en mejorar el sistema de educación pública. Asimismo, el sector público tiene nuevos incentivos para acometer la reforma de los sectores de salud y educación. De ese modo podría iniciarse un círculo virtuoso de una mejor acumulación y distribución de capital humano, ligadas con un crecimiento económico más equitativo. 


\section{I}

\section{Introducción}

En el modelo de desarrollo de comienzos de la posguerra se daba mucha importancia al papel del Estado. El desafío de la coordinación — asegurar la complementación de la inversión pública y privada en los sectores de la industria, el transporte y las comunicaciones necesaria para el despegue de la economíaparecía justificar que el Estado no sólo asumiera el liderazgo como planificador, sino que también tomara la batuta en el manejo de la economía y la producción. En este modelo de desarrollo, gastar en 'sectores no productivos' como la educación y la salud se veía como una carga que obstaculizaba la acumulación de activos productivos $\mathrm{y}$, por lo tanto, como un costo en términos del crecimiento. Los primeros modelos demográficos señalaban, por ejemplo, que el crecimiento acelerado de la población de los países en vías de desarrollo implicaba una desviación de recursos públicos para pagar servicios de educación y salud, con lo que se reducía la disponibilidad de capital físico productivo por habitante.

Los más recientes modelos de crecimiento incorporan el concepto de 'capital humano' como inversión productiva. En los nuevos modelos clásicos de crecimiento, la acumulación de capital humano es un factor tan relevante para el crecimiento como lo era el estrecho concepto de capital físico: se estimula el crecimiento económico aumentando el ahorro y la inversión en educación. Los modelos de crecimiento endógeno más recientes atribuyen una importancia aún mayor al capital humano. En estos modelos, el desarrollo sostenible es posible, en parte, por las externalidades positivas que genera el proceso de educación, importante modalidad del capital humano; para un crecimiento sostenible y elevado son esenciales nuevas ideas y tecnologías, cuyo desarrollo, a su vez, depende de elevados niveles de capital humano.

Los nuevos modelos de crecimiento ofrecen una justificación elegante y poderosa a favor de las inversiones en capital humano por ser eficientes y promovedoras del crecimiento económico. En su forma más simple, sin embargo (como queda de manifiesto en los conocidos estudios empíricos sobre crecimiento de Barro, Sala-i-Martin, Romery otros) incorporan hipótesis poco eficaces para orientar las opciones de política.
En primer lugar, y fundamental para los propósitos de este trabajo, se pasa por alto la distribución del capital humano entre las personas. Se supone en forma implícita que el proceso de acumulación beneficiará, por un proceso de filtración, a los miembros de todos los grupos de ingreso en forma proporcional.

Segundo, y reforzando la primera deficiencia, no se toma en cuenta la demanda. Se considera la acumulación de capital humano como exógena. Los determinantes del proceso de acumulación — decisiones de los hogares de invertir en capital humano y decisiones de política pública respecto del tamaño y asignación de dichas inversiones - no han sido incluidos en los modelos. No se consideran en forma explícita ni la distribución inicial entre los hogares del grado de instrucción de los adultos - factor decisivo para la inversión en educación infantil (Schultz, 1988) — ni las políticas macroeconómicas, comerciales, y otras que abarcan al conjunto de la economía y que también afectan la demanda de educación de los hogares en los distintos grupos de ingresos. Se ignora el hecho de que los padres más pobres y con menor grado de instrucción tienden a invertir menos en la educación de sus hijos.

En tercer lugar, se deja de lado el problema de la entrega: la producción eficiente y equitativa de servicios sociales. Se apoya en forma implícita un papel preponderante del Estado en la provisión de servicios de salud y educación. El acento en externalidades positivas del capital humano en los nuevos modelos de crecimiento se basa en la lógica del fracaso del mercado - especialmente en los mercados de capitalesque inhibe la inversión privada óptima en capital humano. Como el capital humano no se puede apropiar, no puede servir de garantía para préstamos; aun cuando los agentes reconozcan la alta rentabilidad de la inversión privada en salud y educación, no pueden endeudarse, con lo que sus inversiones tienen una fuerte restricción de liquidez. Por ello en estos modelos el papel del Estado es decisivo y refuerza el concepto tradicional del gobierno como principal financista, productor y proveedor de todos los servicios sociales. ${ }^{1}$

\footnotetext{
1 Desde luego, el Estado puede tener un papel importante en el financiamiento selectivo de programas sociales, parte de su provisión, Los nuevos modelos de crecimiento no se pronuncian sobre este punto.
} 
La falta de énfasis en la distribución, la demanda y la entrega de programas sociales ha reforzado la antigua hipótesis de que las sociedades en desarrollo latinoamericanas enfrentan una ineludible compensación de ventajas (trade-off) entre los objetivos de eficiencia y equidad. Siguiendo la tradición de Kuznets, los analistas han tendido a ver la elevada y creciente desigualdad de ingresos en la región como consecuencia inevitable del desarrollo económico. Los esfuerzos de gobiernos en el pasado para rectificar la inequidad mediante transferencias populistas —con desastrosos resultados fiscales - han fomentado el pesimismo respecto de que América Latina pudiera conciliar el crecimiento con la equidad. Dado ese pesimismo, el abanico de recomendaciones de política ha sido relativamente restringido: una política social marginal, diseñada para mitigar el impacto de la creciente pobreza, sin darle ninguna importancia a la inversión en el capital humano de los pobres.

Para rebatir las hipótesis de los nuevos modelos de crecimiento y de los pesimistas del trade off, este artículo se concentra en los sectores sociales de la educación y la salud de América Latina. El proceso de inversión en capital humano en estos dos sectores no ha funcionado bien en la región. Aunque los gobiernos han comprometido tantos o más recursos que otros países en desarrollo en los servicios de salud y educación, los resultados de la región dado su nivel de ingreso son magros. El gasto en estos servicios, especialmente para los pobres, ha sido considerado en el pasado como una transferencia y no como una inversión. La acumulación de capital humano ha sido relativamente lenta, lo que ha afectado negativamente al crecimiento, y ha sido altamente inequitativa entre grupos de ingreso, lo que ha agudizado la desigualdad de los ingresos.

Este artículo busca las razones. La discusión se basa en una consideración más amplia de los puntos mencionados, centrándose en los aspectos interrelacionados de la demanda (de educación, por ejemplo) y de la distribución. Una discusión profunda del tercer punto, la transformación de la entrega de los servicios sociales, puede encontrarse en Birdsall y Londoño (1998).

En primer lugar, en lo que toca a la demanda, la baja acumulación de capital humano en América Latina refleja la escasa demanda de educación de los hogares, especialmente de los más pobres. Por otro lado, en cuanto a la distribución, la baja demanda de capital humano de los hogares está ligada a la elevada proporción de hogares pobres en América Latina y a la profundidad de su pobreza. En América Latina, el acceso históricamente inequitativo de los pobres a los activos que generan renta - tanto tierra como capital humano - ayuda a explicar el círculo vicioso de la baja acumulación de capital humano y la pobreza. Más aún, la escasa acumulación de capital humano de los pobres se ha acentuado por padrones regresivos del gasto público en programas sociales - los pobres no se han beneficiado mucho del gasto público en servicios de salud y educación - y por las políticas económicas que han penalizado al trabajo y han desalentado la inversión de los hogares en educación.

En resumen, en América Latina la teoría de la filtración (trickte-down) no ha sido aplicable a la acumulación de capital humano. Si las economías de la región han de aprovechar los efectos de eficiencia y promoción del crecimiento de una más rápida acumulación de capital humano, deben darle mayor importancia a la equidad en la distribución de los servicios que generan capital humano. ${ }^{2}$

El artículo describe, para comenzar, la naturaleza del desafío, resumiendo datos que demuestran que, a pesar de un nivel adecuado de gasto público, la acumulación de capital humano en América Latina ha sido baja e inequitativa - la distribución de la educación apenas si ha mejorado en el transcurso del tiempo. El análisis empírico muestra que la acumulación baja e inequitativa del capital humano explica en gran parte no sólo el alto nivel de pobreza y la disparidad de ingresos en América Latina, sino también su lenta formación de capital y la falta de crecimiento económico; y que, en un círculo vicioso, la insuficiencia y !a desigualdad de los recursos humanos de la región han sido efecto y al mismo tiempo factor coadyuvante del alto grado de desigualdad de activos e ingresos en toda América Latina.

Después se analizan las razones de esta baja y desigual acumulación de capital humano; se destaca el efecto de una débil demanda de educación entre los pobres debido a restricciones de liquidez y la probabilidad de una baja rentabilidad de la inversión en capital humano en las economías sesgadas contra el trabajo. Por una parte, la historia sugiere un desalentador círculo vicioso en el que la pobreza y la desigualdad de ingreso iniciales se han traducido en una acumula-

\footnotetext{
2 En Birdsail y Londoño (1998) planteamos el problema de una entrega más equitativa de servicios sociales mediante un nuevo enfoque horizontal a la provisión de servicios sociales, que descansa en una mayor focalización del gasto público en los pobres, una oferta de servicios más competitiva y una demanda que fortalece el poder de los consumidores, incluidos los pobres.
} 
ción de capital humano lenta y desigual, que a su vez ha reducido el crecimiento económico y acentuado la desigualdad. Por otra parte, hay una consecuencia positiva: una acumulación más rápida, con mayor énfasis en el acceso igualitario a la educación, puede acelerar el crecimiento económico y reducir la desigualdad de los ingresos. Más educación y una educación más igualitaria pueden promover tanto la eficiencia como la equidad.

Este artículo concluye con una nota de optimismo. Las recientes reformas generales de la economía están aumentando la demanda de mano de obra calificada en la región. Ello eleva la rentabilidad de la inversión en capital humano y genera una nueva ola de interés del sector empresarial por contar con un mejor sistema de educación pública. Al mismo tiempo, el éxito en el frente macroeconómico ha generado una disposición en el sector público para emprender refor- mas en los sectores de salud y educación: la educación fue tema principal de atención de los jefes de Estado en la Cumbre de las Américas de Santiago de Chile (1998). En toda la región se están ensayando numerosos nuevos sistemas de provisión de servicios sociales que combinan las reglas del juego impuestas centralmente con la oferta competitiva del mercado y el fortalecimiento del poder de los consumidores. Estos intentos muestran que es posible una mayor eficiencia interna en la provisión de servicios sociales y una mayor capacidad de respuesta y responsabilidad de los sistemas públicos frente a las necesidades de los pobres. Un mayor acceso de los pobres a buenos servicios de educación y salud puede poner en movimiento un nuevo círculo virtuoso que incluya una distribución más justa de activos y oportunidades, un crecimiento económico más rápido y una distribución más amplia de sus beneficios.

\section{II}

\section{La baja y desigual acumulación de capital humano en América Latina}

Examinemos en primer lugar cuáles son los hechos. Cotejado con su ingreso por habitante, el proceso de acumulación de capital humano en América Latina es débil en comparación con otras regiones. Este hecho es más evidente en el sector de la educación: la escolaridad media es inferior en dos años a la que le corresponde por su ingreso por habitante, apenas supera el desempeño del África subsahariana y está muy por debajo de la que se observa en Asia oriental y sudoriental. A principios del decenio de 1990, los trabajadores tenían un promedio de 5.2 años de escolaridad, casi un tercio menos de lo que podía esperarse para países con un nivel de desarrollo similar; más de un tercio de los niños que entraban a la escuela primaria no la terminaban, tasa de deserción que más que duplica la de otras regiones del mundo.

La brecha educacional ha empeorado en los últimos treinta años. A comienzos de los años setenta, América Latina tenía un nivel de instrucción más bajo que países de niveles de ingreso comparables de Europa y el sudeste asiático, pero equiparable al de los demás países en desarrollo, si se lo medía por habitante. Desde entonces, la educación en América Latina (encabezada por Brasil, México, Venezuela y Améri- ca Central) ha crecido a una tasa positiva pero lenta, muy por debajo de las tasas registradas por los países asiáticos y el resto del mundo en desarrollo. En 1980, la fuerza trabajadora de la región iba atrasada en un año con respecto al promedio de los países en desarrollo en cuanto al número de años cursados. A mediados del decenio de 1990, este atraso se había duplicado. En comparación con Asia oriental y sudoriental, el déficit educativo de América Latina subió de menos de un año, en 1970, a alrededor de cuatro en $1995^{\circ}$ (gráfico 1).

El déficit en salud es más pequeño en comparación con otras regiones. La esperanza media de vida es casi dos años menor que la correspondiente a su nivel de ingreso (gráfico 2). Es posible que ello sea consecuencia de una menor diferencia que en otras regiones en cuanto a género en la educación, al efecto positivo del grado de instrucción de las madres en la mortalidad infantil y al gasto e innovación relativamente mayores en el sector de salud en América Latina (BID; 1996).

El problema básico no es el de un bajo nivel de gasto en salud y educación —el que es similar al de otras regiones en desarrollo, con un $6.6 \%$ del PIB (cua- 
GRÁFICO 1

\section{América Latina y Asia oriental:} El déficit educativo

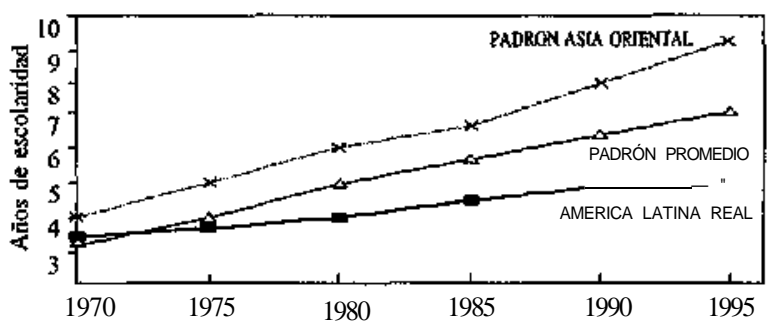

Fuente: Banco Interamericano de Desarrollo, Progreso económico y social en América Latina, Washington D.C., 1996.

dro 1$)^{3}$-, sino más bien de utilización ineficiente del gasto público y de distribución poco equitativa de la incidencia del gasto en esos servicios, es decir, la proporción relativamente baja de ese gasto que ha beneficiado a los pobres. ${ }^{4}$ En comparación con Asia oriental y los países industrializados, América Latina muestra una alta desigualdad en la distribución de su capital humano. (La desigualdad en la distribución de la tierra, otro activo productivo crítico, es la más alta del mundo - véanse los gráficos 3 y 4), ${ }^{5}$ Por lo tanto, la baja tasa de acumulación general - un promedio de grandes aumentos de escolaridad para unos pocos y escasos aumentos para la gran mayoría - es produc-

3 Igual que los resultados, el gasto en salud es relativamente más elevado que en educación en comparación con otras regiones. En este artículo se destaca la educación como medida de acumulación de capital humano. La salud es otra inversión en capital humano, pero con efectos menos mensurables - y, por lo tanto, más difíciles de medir y diferenciar - en la productividad y el crecimiento del ingreso.

4 En comparación con Asia oriental, el bajo crecimiento económico y la posterior caída de la fecundidad se tradujeron también en una disminución del gasto por niño en América Latina, especialmente durante el decenio de 1980. Ahora América Latina está empezando a beneficiarse de una reducción del crecimiento de la población en edad escolar y de un aumento absoluto del gasto por habitante que se asocia a lasas de crecimiento económico más elevadas, aun si no aumenta la proporción del PIB destinado a gasto social. Además, en muchos países esa proporción también está aumentando.

5 En los gráficos 3 y 4, se estiman los años de escolaridad usando la distribución de frecuencias de Barro-Lee para la población de 25 años y más entre las categorías de escolaridad ('sin escolàridad', 'primaria incompleta', 'primaria completa', 'secundaria incompleta', 'secundaria completa', 'superior incompleta' y 'superior completa'), Hay datos que muestran que en América Latina estaría disminuyendo la desigualdad de la educación: la desigualdad era menor para jos adultos jóvenes (entre 20 y 30 años) que para grupos mayores en 1990 (Elizabeth King, correspondencia personal, junio de 1997). Sin embargo, la experiencia de países como indonesia muestra que aun a partir de niveles bajos, se puede asociar la acumulación rápida con mejoramientos en la distribución.
GRÁFICO 2

\section{América Latina: El déficit de la esperanza de vida}

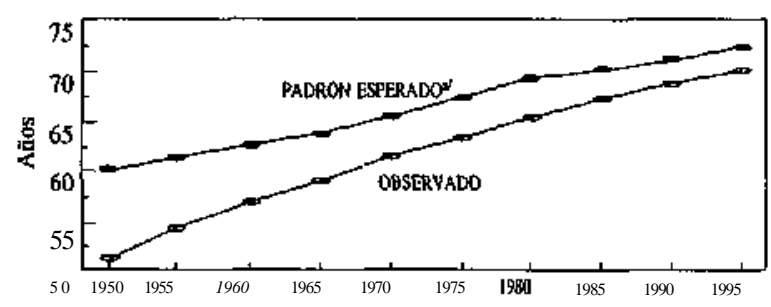

Fuente: Banco Interamericano de Desarrollo, Progreso económico y social en América Latina, Washington D.C, 1996.

a Calculado de la ecuación e(ln (y), time).

CUADRO 1

Gasto social en el decenio de 1990 (Por ciento del PIB)

\begin{tabular}{|c|c|c|c|c|}
\hline & \multicolumn{3}{|c|}{ Gasto público } & \multirow{2}{*}{$\frac{\text { Gasto privado }}{\text { Salud }}$} \\
\hline & Total & Educación & Salud & \\
\hline \multirow{3}{*}{$\begin{array}{l}\text { América Latina } \\
\text { Otros países en desarrollo } \\
\text { Todos los países en } \\
\text { desarrollo }\end{array}$} & 6.6 & 3.6 & 3.0 & 3.1 \\
\hline & 6.4 & 4.2 & & 1.9 \\
\hline & 6.5 & 1.1 & 2.2 & 2.2 \\
\hline Total mundial & 9.9 & 5.1 & & 3.2 \\
\hline
\end{tabular}

Fuente: Banco Interamericano de Desarrollo (BID), Progreso económico y social en América Latina, Washington, D.C, 1996.

to, en parte, de la naturaleza desigual de la acumulación. ${ }^{6}$

Además, y contrariamente a lo que cabría esperar, el aumento de la escolaridad media en América Latina en los últimos treinta años no ha estado asociado con el mejoramiento de la distribución de la educación. En el gráfico 5 se compara la distribución prác-

\footnotetext{
${ }^{6}$ La baja acumulación en América Latina se asocia estrechamente con la mala calidad de las escuelas públicas primarias y secundarias, como describen y analizan Schiefelbein, 1995 y Birdsall, 1998. Entre una multitud de problemas sistémicos de los sistemas altamente centralizados está la dificullad de contratación y retención de los mejores profesores. En muchos países los profesores son muy mal pagados; más aún, los profesores, incluyendo a los menos preparados, son mal apoyados, pero sin embargo avanzan automáticamente en el sistema persistiendo a menudo en prácticas laborales insatisfactorias para beneficiarse de pensiones garantizadas y para retirarse a tan temprana edad como a los 50 años. Muchos presupuestos están recargados de pagos a profesores inactivos, ya sea retirados o profesores "fantasmas", lo que merma los recursos disponibles para los profesores activos. Es un desafío para los gobiernos de la región crear mecanismos para atraer la colaboración y la cooperación de los gremios de profesores, algunos de los cuales están politizados y se resisten al cambio.
} 
GRÁFICO 3

Desigualdad de distribución

de los activos, alrededor de 1990

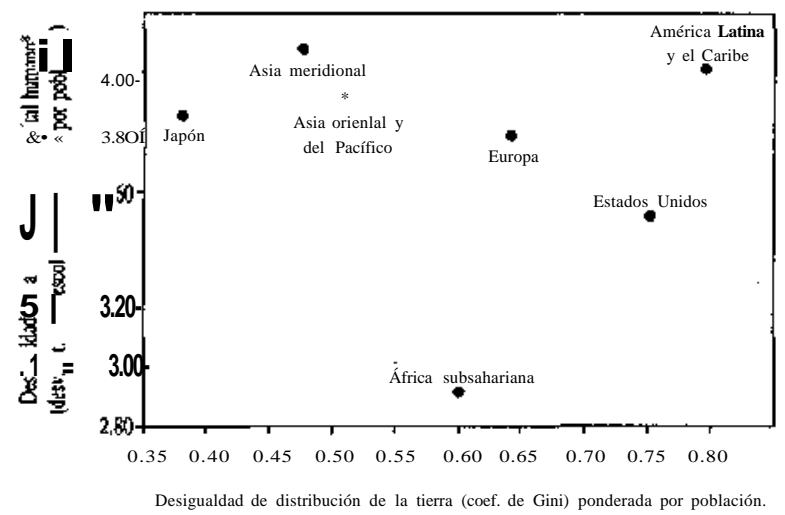

Fuente: La desigualdad de distribución del capital humano se calculó utilizando cifras de educación de R.J. Barro y J. Lee, International Comparison of Educational Attainnent, Washington D.C., Banco Mundial, 1993. Los coeficientes de Gini para la distribución de la tierra se obtuvieron de K, Deininger y L. Squire (correspondencia personal).

a La medida de desigualdad de distribución del capital humano es la desviación estándar de la escolaridad de la población de 25 años y más (véase la nota de pie de página número 5).

GRÁFICO 5

América Latina y el Caribe y Asia oriental: Desigualdad de distribución del capital humano, una comparación regional

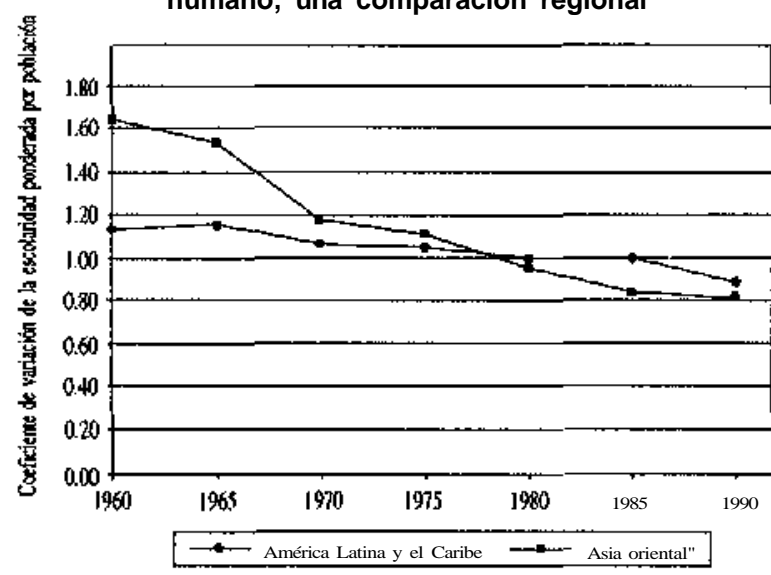

Fuente: La desigualdad de la distribución del capital humano se calculó con la información de escolaridad de R.J. Barro y J. Lee, International Comparisons in Educational Attainment, Washington D.C., Banco Mundial, 1993.

a En Asia oriental se incluyen Hong Kong, Indonesia, la República de Corea, Malasia, Singapur, Taiwán y Tailandia. La desigualdad de distribución del capital humano se mide aquí por el coeficiente de variación de la escolaridad.
GRÁFICO 4

\section{Desigualdad de distribución de los activos, alrededor de $1990^{\mathrm{a}}$}

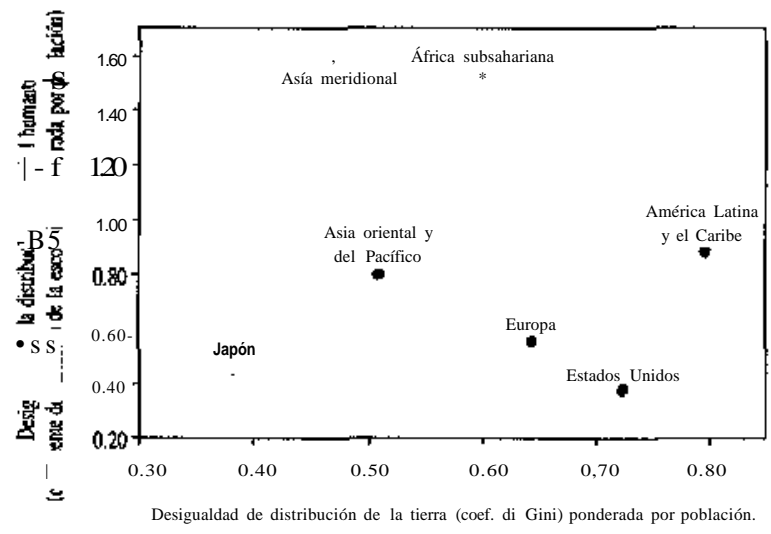

Fuente: La desigualdad del capital humano se calculó utilizando las cifras de educación de R.J. Barro y J. Lee International Comparison of Educational Attainment, Washington D.C., Banco Mundial, 1993. Los coeficientes de Gini para la tierra se obtuvieron de K. Deininger y $L$. Squire (correspondencia personal).

a La medida de desigualdad del capital humano es el coeficiente de variación, es decir, el cuociente entre la desviación estándar y la media; esta medida considera el efecto de cambios en el promedio de escolaridad en la distribución (véase la nota de pie de página número 5). Nótese que la alta desigualdad del capital humano en América Latina (gráfico 3) se reduce en relación con otras regiones al dividir la desviación estándar de la escolaridad de adultos por la media de la escolaridad de adultos. El nivel relativamente alto de escolaridad en América Latina en comparación con otros pafses en desarrollo no es suficiente para compensar la elevada desviación estándar para una medía dada, en comparación con Asia oriental y las regiones desarrolladas.

ticamente estática de la educación en América Latina con el mejoramiento en Asia oriental durante este período. $^{7}$

\section{Efectos de la acumulación baja y desigual so- bre el crecimiento}

¿Cuáles han sido los efectos en la tasa de crecimiento económico de la baja y desigual acumulación de capital humano en América Latina en los últimos treinta años? El cuadro 2 muestra los resultados de la estimación de una ecuación de crecimiento tradicional para los países de la región, utilizando la mejor y más reciente información disponible sobre distribución del ingreso (Deininger y Squire, 1996). Para estas estima-

\footnotetext{
${ }^{7}$ Es normal que las disparidades en el capital humano de la población aumenten durante la expansión inicial de los sistemas educacionales, hasta que el grado medio de instrucción llegue a los cinco o seis años (Londoño y Székely, 1997).
} 
ciones se seleccionaron los países en que se disponía de una curva de Lorenz para dos períodos con una separación de al menos cinco años entre ellos, de estimaciones de ingreso por habitante a precios de poder de compra internacionales y de información sobre inversión en capital físico, escolaridad de la fuerza trabajadora (que se utilizó para medir la distribución del capital humano), distribución de la tierra e indicadores de comercio.

Las conclusiones (dadas a conocer originalmente en Birdsall y Londoño, 1997) eran claras respecto del efecto de la educación y su distribución sobre el cre- cimiento económico. La acumulación de educación, así como la acumulación de capital, es buena para el crecimiento económico — un resultado que ahora se da por sentado (columnas 2 y 3). El gran efecto positivo de la acumulación de capital humano en el crecimiento económico, como lo muestra el promedio de escolaridad de la fuerza trabajadora, es concordante con la teoría mencionada en la introducción, así como con datos microeconómicos que muestran que los trabajadores con mayor nivel de instrucción ganan ingresos más altos y, particularmente las mujeres, son más eficaces en la producción familiar de buena salud y es-

CUADRO 2

Explicación del crecimiento

\begin{tabular}{|c|c|c|c|}
\hline $\begin{array}{l}\text { A. Explicación del crecimiento global } \\
\text { Variable independiente }\end{array}$ & (1) & (2) & (3) \\
\hline Constante & 0.01 & $0.04^{\mathrm{a}}$ & 0.03 \\
\hline Acumulación de capital & $0.53^{\mathrm{a}}$ & $0.57^{\mathrm{a}}$ & $0.54^{! 1}$ \\
\hline \multicolumn{4}{|l|}{ Condiciones iniciales: } \\
\hline Nivel de ingreso & -0.88 & -0.41 & -0.42 \\
\hline Nivel de educación & 0.17 & $0.28^{\mathrm{h}}$ & $0.30^{\mathrm{h}}$ \\
\hline Desigualdad en distribución del ingreso & $-0.05^{\mathrm{b}}$ & -0.03 & -0.002 \\
\hline Desigualdad en distribución de la tierra & & $-0.02^{1 \prime}$ & $-0.0 \mathrm{I}$ \\
\hline Desigualdad en distribución de la educación & & $-0.09^{b}$ & $-0.09^{b}$ \\
\hline Recursos naturales & & & -0.01 \\
\hline \multicolumn{4}{|l|}{ Cambios en: } \\
\hline \multicolumn{4}{|l|}{ Desigualdad en distribución del ingreso } \\
\hline & & & 0.02 \\
\hline \multicolumn{4}{|l|}{ Comercio de bienes manufacturados } \\
\hline \multicolumn{4}{|l|}{ Comercio de bienes primarios } \\
\hline \multicolumn{3}{|l|}{ Variable ficticia (dummy) para América Latina y el Caribe } & 0.004 \\
\hline $\mathbf{R}^{2}$ & 0.61 & 0.70 & 0.76 \\
\hline \multicolumn{4}{|l|}{ B. Explicación del aumento del ingreso de los más pobres } \\
\hline Variable independiente & (4) & (5) & (6) \\
\hline Constante & 0.00 & $0.05^{\mathrm{C}}$ & $0.04^{\circ}$ \\
\hline Crecimiento global & $1.31^{\mathrm{a}}$ & & \\
\hline Acumulación de capital & & $0.72^{\mathrm{a}}$ & $0.77^{\mathrm{a}}$ \\
\hline \multicolumn{4}{|l|}{ Condiciones iniciales: } \\
\hline \multicolumn{4}{|l|}{ Nivel de ingreso } \\
\hline Nivel de educación & & $0.4 \mathrm{l}^{\mathrm{c}}$ & $0.51^{\mathrm{c}}$ \\
\hline Desigualdad en distribución del ingreso & & 0.05 & 0.02 \\
\hline Desigualdad en distribución de la tierra & & $-0.07^{1}$ & -0.02 \\
\hline Desigualdad en distribución de la educación & & $-0.20^{1 "}$ & $-0 . \mathbf{I}^{\mathrm{b}}$ \\
\hline \multicolumn{4}{|l|}{ Recursos naturales } \\
\hline \multicolumn{4}{|l|}{ Cambios en: } \\
\hline Desigualdad en distribución del ingreso & & & $-0.27^{\mathrm{a}}$ \\
\hline \multicolumn{4}{|l|}{ Apertura del comercio } \\
\hline Comercio de bienes manufacturados & & & $0.05^{\mathrm{e}}$ \\
\hline Comercio de bienes primarios & & & -0.01 \\
\hline Variable ficticia (dummy) & & & -0.01 \\
\hline $\mathbf{R}^{2}$ & 0.51 & 0.42 & 0.63 \\
\hline
\end{tabular}

Fuente: N. Birdsall $y$ J.L. Londoño, Asset inequality matters: an assessment of the World Bank's approach to poverty reduction, The American Economic Review; vol. 87, 수 2, Nashville, Tennessee, American Economic Association, 1997,

a Estadísticamente significativo a nivel de 1 por ciento.

${ }^{\mathrm{b}}$ Estadísticamente significativo a nivel de 5 por ciento.

c Estadísticamente significativo a nivel de 10 por ciento. 
colaridad de los niños. El efecto positivo del grado de instrucción del país al comienzo de un período sobre el crecimiento en el período siguiente es hoy un lugar común y conclusión muy firme de casi todos los estudios sobre el crecimiento económico (Barro y Sala-iMartin, 1995; Levine y Renelt, 1992). Lora y Barrera (1997) estiman que América Latina como región podría aumentar su tasa de crecimiento en 2 puntos porcentuales anuales en la próxima década si, además de profundizar las reformas estructurales, fuera capaz de acelerar el ritmo de acumulación de capital humano para el conjunto de la fuerza de trabajo adelantándose en un año a la tendencia esperada. Visto desde otra perspectiva, Birdsall, Ross y Sabot (1995) estiman que Corea, con el nivel de educación primaria y secundaria que Brasil tenía en 1960, habría crecido 0.56 puntos menos por año en los 25 años siguientes, y que el crecimiento del PIB por habitante de Corea habría sido en 1985 un $12 \%$ inferior a lo que fue en realidad.

Además, y controlando por el nivel de instrucción, el grado de desigualdad en la distribución de la educación tiene un fuerte efecto negativo sobre el crecimiento (columnas 2 y 3). La variable que mide la distribución de la educación es muy confiable $;^{8}$ su efecto negativo es independiente no sólo de la variable escolaridad, sino del efecto positivo de la apertura del comercio y el negativo de la dotación natural de recursos.

Nótese que cuando se incluyen las variables de distribución de activos el efecto negativo de la desigualdad de ingresos en el crecimiento económico pierde significación estadística (columnas 2 y 3 comparadas con columna 1); el efecto negativo tantas veces comentado (Birdsall, Ross y Sabot, 1995; Alesina y Rodrik, 1994; Persson y Tabellini, 1994) aparentemente corresponde a diferencias en un elemento fundamental de la estructura económica, a saber, el acceso de los diferentes grupos a los activos productivos.

En las columnas 4 a 6 del cuadro 2 , se evalúa el efecto de las distribuciones iniciales de ingresos y activos sobre el crecimiento del ingreso de los pobres. La elasticidad del crecimiento del ingreso de los pobres respecto al crecimiento general es muy superior a 1 (columna 1), lo que confirma la lógica del argumento de que el crecimiento económico es clave para la reducción de la pobreza. El aumento del ingreso de

\footnotetext{
8 En estas ecuaciones de regresión se utiliza como medida de la distribución de la educación la desviación estándar de años de escolaridad de los adultos de 25 años o más.
}

los pobres depende fuertemente también de la acumulación de capital (columnas 2 y 3). Es interesante ver que las desigualdades iniciales en la distribución de la tierra y del capital humano tienen un claro impacto negativo en el crecimiento del ingreso de los pobres, de magnitudes que duplican sus efectos en el crecimiento del ingreso medio (columna 2). Una distribución desigual de los activos, especialmente del capital humano, afecta el crecimiento del ingreso de los pobres en forma desproporcionada; una mejor distribución de los activos reduciría la pobreza en forma directa e indirecta, al impulsar el crecimiento medio.

Al plantear este argumento con indicadores económicos globales, Birdsall y Londoño (1997) demuestran que si las economías de América Latina hubieran mantenido la misma distribución del ingreso del decenio de 1970 en el de 1980, el aumento de la pobreza entre 1983 y 1995 habría sido casi la mitad (gráfico 6). Pero el crecimiento lento y la inestabilidad macroeconómica del decenio de 1980 hicieron más daño a los pobres que a otros grupos, agudizando una mala distribución de los activos y del ingreso. Estos resultados coinciden con una visión del mundo en que las oportunidades sí importan. Los pobres, sin activos, no pueden aprovechar las oportunidades de ser productivos. En economías en que una parte importante de la población carece de capital humano, y por lo tanto de un activo productivo decisivo, sólo una fracción de la población puede explotar el proceso de crecimiento. El motor del crecimiento es pequeño y puede atascarse de cuando en cuando con rodeos populistas. Impulsados

GRÁFICO 6

América Latina: Impacto de la desigualdad en la pobreza, 1970-1995

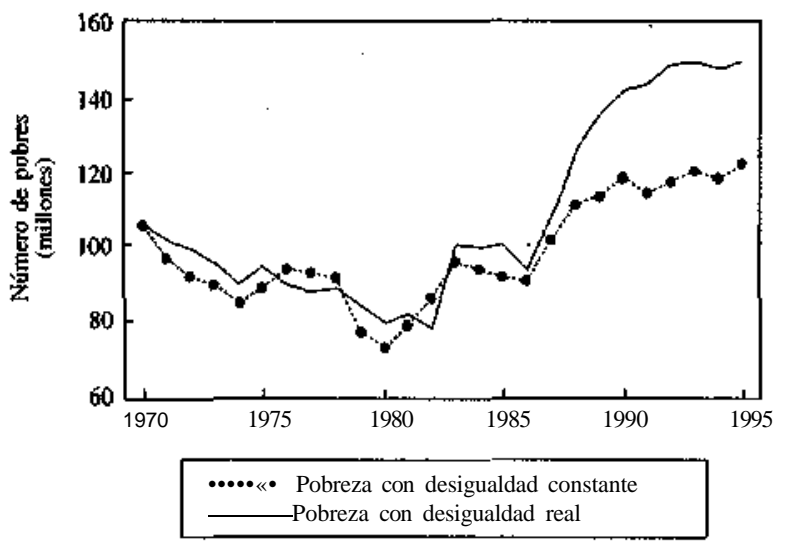

Fuente: N. Birdsall y J.L. Londoño, Asset inequality matters: An assessment of the World Bank's approach to poverty reduction. The American Economic Review, vol. 87, № 2, Nashville, Tennessee, American Economic Association, 1997. 
GRÁFICO 7

\section{Algunas regiones: Factores del} crecimiento global

(Contribución a las variaciones del PIB, como porcentaje)

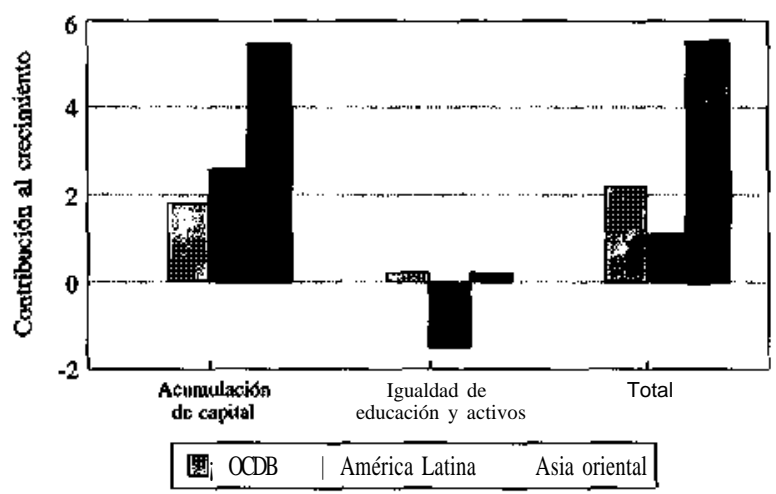

Fuente: Banco Interamericano de Desarrollo.

por la creciente productividad de los pobres iniciales, los países del Asia oriental, que comenzaron el período de posguerra con una desigualdad de activos relativamente baja, fueron capaces de crecer a tasas altas y sostenidas por más de tres décadas. En cambio, la mayoría de los países de América Latina, con mayor desigualdad de activos, y presumiblemente con menos oportunidades para sus pobres, crecieron menos (gráfico 7). Los resultados del cuadro 2 y los indicadores globales reseñados apuntan a una conclusión tan clara como inquietante: la baja y desigual acumulación de capital humano en América Latina ha retardado el crecimiento económico global y ha inhibido la reducción de la pobreza.

\section{Efectos sobre la desigualdad del ingreso}

Un segundo efecto de la baja y desigual acumulación es la desigualdad persistentemente alta de los ingresos en América Latina.

El coeficiente de Gini de América Latina (cerca de 0.50 para el conjunto de la región) es aproximadamente 15 puntos más alto que el promedio para el resto del mundo; en 1995, el 20\% más rico de la población recibía el $58 \%$ del ingreso total, es decir, 12 veces el ingreso del $40 \%$ más pobre de la población y 19 veces el ingreso del $20 \%$ más pobre. El ingreso del $40 \%$ más pobre de la población es unas 20 veces más bajo que lo que le correspondería con un padrón distribución típico del ingreso. Y el número de pobres, que hoy se calcula entre 140 y 150 millones de personas con ingresos diarios inferiores a 2 dólares, podría reducirse en un tercio (Londoño y Székely, 1997).

Londoño y Székely (1997) muestran que la desigualdad de ingresos en distintos países del mundo se explica razonablemente por la abundancia relativa de factores de producción, como la tierra y el capital humano, y por su distribución. Como se aprecia en el gráfico 8, la menor acumulación de capital físico no explica la desigualdad en el caso en América Latina. Por el contrario, la abundancia relativa de recursos naturales y la mayor concentración de la tierra en el mundo son la causa de una gran parte de la excesiva desigualdad de la región. El bajo nivel de escolaridad (capital humano) de los trabajadores latinoamericanos y la enorme desigualdad en los activos educativos forman también gran parte de la explicación.

No sorprende el efecto de la baja y desigual educación sobre la desigualdad del ingreso. Donde una proporción relativamente escasa de la población completa la educación secundaria o superior, la inversión pública en educación es considerada por lo general como un mecanismo para reducir la pobreza y la desigualdad, dada la poderosa demostración a nivel individual de que los de mayor grado de instrucción ganan más. A nivel global, sin embargo, el efecto de la educación en la pobreza y la desigualdad depende obviamente de la distribución de la educación misma, de la rapidez de su expansión y de cómo se benefician de ella los distintos grupos. En América Latina sólo una proporción reducida de la población ha terminado

GRÁFICO 8

América Latina: Explicación del exceso de desigualdad en esta región en comparación con el promedio mundial

(Separado por composición, nivel y distribución de los activos)

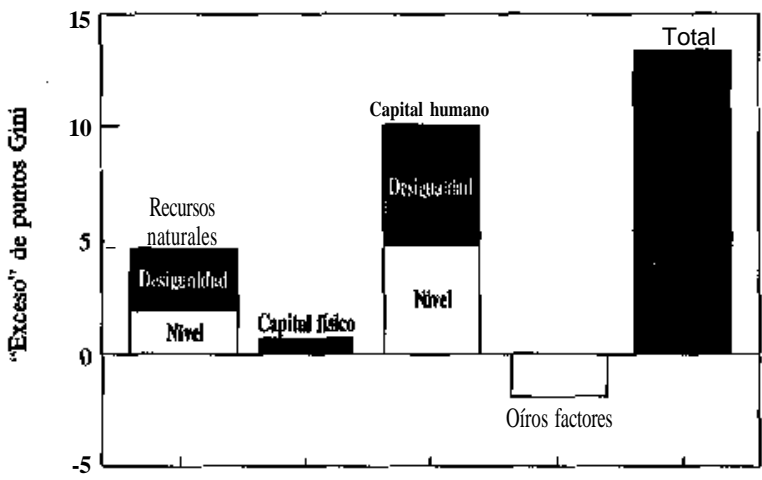

Fuente: J.L. Londoño y M. Székely, Distributional surprises after a decade of reforms: Latin America in the nineties, en R. Hausman y E. Lora (comp.), Latin America after a Decade of Reforms: What comes next?, Washington D.C., BID, 1997. 
la educación secundaria o superior. Estos trabajadores calificados, relativamente poco numerosos, ganan un salario mucho más alto por lo limitado de su oferta y contribuyen así a la gran desigualdad general de los ingresos (Birdsall, Ross y Sabot, 1997).

La experiencia latinoamericana contrasta abiertamente con la del Asia oriental, donde la política educacional ha producido un gran número de trabajadores calificados, lo que ha disminuido cualquier gran diferencial que pudiera haber habido en comparación con los salarios de la mano de obra no calificada. La teoría de Kuznets (de que la distribución del ingreso se deteriorará inicialmente mientras los trabajadores de las economías subdesarrolladas se trasladan a sectores más productivos) parece confirmarse en América Latina, en parte porque la expansión limitada y lenta de las oportunidades educativas ha generado una gran brecha de productividad entre un grupo pequeño de trabajadores calificados y el resto de la población (Stallings, Birdsall y Clugage, por publicarse).

\section{III}

\section{Las razones de la acumulación baja y desigual en América Latina}

El análisis anterior muestra que la acumulación baja y desigual de capital humano ha limitado el crecimiento económico de América Latina, especialmente en el ingreso de los pobres, y ha agudizado la ya elevada desigualdad de la distribución del ingreso en la región. Ahora sostenemos que, de hecho, hay un círculo vicioso: la acumulación lenta y desigual de capital humano en la región no es sólo causa de la actual pobreza y desigualdad, sino también consecuencia de la desigualdad anterior. La acumulación baja y desigual se puede explicar por factores de oferta y demanda en el mercado de la educación. ${ }^{9}$

En primer lugar, por el lado de la demanda, la gran dotación de recursos naturales de América Latina ha limitado históricamente la demanda de educación de la sociedad. La organización socioeconómica que acompaña a la producción agrícola extensiva y a la extracción de recursos naturales tiende a caracterizarse por relativamente pocos propietarios del capital y un gran número de trabajadores no calificados (Engerman y Sokoloff, 1997). Hay poca demanda de trabajadores calificados, en parte porque los recursos naturales tienden a ser complementarios del capital, no del trabajo calificado, en la producción. Quizás como resultado los gobiernos y los hogares latinoamericanos han invertido poco en educación, persiguiendo la mayor rentabilidad del capital físico. Una base rica en recursos naturales minimizó también la necesidad de desarrollar

\footnotetext{
${ }^{9}$ La discusión y los datos de esta sección están basados en Birdsall, Bruns y Sabot (1996).
}

exportaciones no tradicionales competitivas a comienzos del período de posguerra, perpetuándose de esta manera la organización tradicional de la producción.

En segundo lugar, la alta desigualdad de ingresos en América Latina implica que un mayor número de hogares carecen de la liquidez necesaria, no son capaces de endeudarse y no disponen de los recursos para mantener a sus niños en la escuela. Flugg, Spilimbergo y Watchtenheim (1996) muestran que el factor financiero explica muchas de las diferencias de escolaridad secundaria entre los países. Como se aprecia en el cuadro 3, en 1989 Brasil y Malasia tenían niveles similares de ingreso por habitante. Pero el quintil más pobre de Brasil tenía alrededor de la mitad del nivel de ingreso absoluto del quintil más pobre de Malasia. Para una elasticidad ingreso de la demanda de educación secundaria de 0.50 (cifra conservadora), si la distribución del ingreso hubiera sido tan igual en Brasil como en Malasia, la matrícula secundaria de los niños brasileños pobres habría sido un $40 \%$ más alta. Hay datos que demuestran que, entre los pobres, la elasticidad ingreso de la demanda de educación básica es mayor que 1.0 , en cuyo caso la matricula secundaria de los niños pobres del Brasil habría subido en más de un $80 \%$. Un estudio cuantitativo sobre el efecto de la desigualdad del ingreso en la escolaridad sugiere que, de los 27 puntos porcentuales de diferencia de matrícula secundaria entre Brasil y Corea en el decenio de 1970, más de 20 podían atribuirse a la mayor desigualdad de ingresos del Brasil, que se traduce en una menor matricula de los niños pobres (Williamson, 1993). 
CUADRO 3

Participación absoluta en el ingreso del quintil más bajo

\begin{tabular}{lccc}
\hline País & $\begin{array}{c}\text { PIB por habitante } \\
\text { (dólares de igual poder } \\
\text { adquisitivo) }\end{array}$ & $\begin{array}{c}\text { Participación en el ingreso del 20\% } \\
\text { de los hogares más pobres } \\
(\%)\end{array}$ & $\begin{array}{c}\text { Ingreso por habitante del 20\% } \\
\text { de los hogares más pobres } \\
\text { (dólares) }\end{array}$ \\
\hline $\begin{array}{l}\text { Malasia, 1989 } \\
\text { Brasil, !989 }\end{array}$ & 4674 & 4.6 & 1075 \\
\hline
\end{tabular}

Fuente: Para PIB, R. Summers y A. Heston, The Penn World Tables, Mark 5.6, Philadelphia, Pa., University of Pennsylvania, Department of Economics , 1995; para participación en el ingreso, K. Deiningery L. Squire, A new data set measuring income inequality, The World Bank Economic Review, vol. 10, N 3, Washington D.C., Banco Mundial, 1996.

En tercer lugar, la demanda de educación de los hogares no es sólo función del ingreso del hogar y de su acceso al crédito. También es función de la rentabilidad que espera la familia de la escolaridad, en términos de los ingresos futuros de los niños escolarizados. Dos políticas públicas diferentes han reducido en forma sistemática la demanda de educación básica entre los pobres al reducir la rentabilidad prevista.

Por un lado, las estrategias económicas han castigado a la mano de obra reduciendo su rentabilidad y desalentando la inversión en educación. Los gobiernos latinoamericanos de la posguerra llevaron a cabo políticas de industrialización mediante sustitución de importaciones en un intento de apartarse de las exportaciones de bienes primarios y como una forma de promover la manufactura local. Estas políticas de industrialización sustitutiva se tradujeron en grandes subvenciones y protección a los dueños del capital sin promover la demanda de trabajo (Schiff y Valdés, 1992), Como resultado, subieron las ganancias de los dueños del capital y en menor proporción los salarios reales de los trabajadores no calificados. El bajo crecimiento de los salarios relativos de los trabajadores y la elevada rentabilidad del capital no estimularon la demanda de educación básica entre los pobres. En la última década, la mayoría de los países de la región ha abandonado estas políticas en la búsqueda de estrategias de crecimiento de mercado abierto. En algunos casos, sin embargo, una preocupación atendible por reducir la inflación ha hecho necesario sostener los tipos de cambio con tasas de interés elevadas; esto ha castigado la creación de empleos en el sector de la empresa mediana y pequeña y ha disminuido las exportaciones que con frecuencia hacen uso más intensivo de mano de obra.

Además, en algunas partes de América Latina ha habido discriminación contra determinados grupos étnicos, lingüísticos o raciales que tienden también a ser más pobres. Esta discriminación ha reducido la rentabilidad de la educación entre ellos y deprimido aún más la demanda de educación entre los pobres.

Por su parte, la política educativa en sí ha sido un problema. La mala calidad de la educación básica en América Latina y su deterioro, producto de un gasto público ineficiente, ha reducido la rentabilidad de la escolaridad básica en la región, especialmente para los hogares, pobres cuyos niños tienen una mayor probabilidad de asistir a las peores escuelas. Las altas tasas de repetición y deserción escolares de América Latina, especialmente entre los pobres, son un triste testimonio del esfuerzo inicial de los padres para matricular a sus hijos y de su creciente desaliento cuando la baja calidad de la enseñanza limita el aprendizaje y, con ello, reduce la rentabilidad económica esperada. ${ }^{10}$

En resumen, la rentabilidad esperada de la educación es función de la evaluación de los padres del mercado laboral futuro para sus hijos. Si la demanda de trabajo es pequeña (y el capital se subvenciona directa o indirectamente), si la enseñanza es de mala calidad y si existe discriminación en el mercado de trabajo contra algunos grupos que también tienden a ser pobres, la baja rentabilidad de la educación escolar va a reducir la demanda de educación de los hogares.

Al mismo tiempo, la oferta de educación en América Latina se ha visto afectada por la alta desigualdad de ingresos de la región." Cuando la distribución del ingreso es altamente desigual, entregar educación bá-

\footnotetext{
10 Las causas de la baja calidad de la educación básica y sus consecuencias entre los pobres se abordan ampliamente para Brasil en Birdsall, Bruns y Sabot (1996) y para América Latina en Birdsall y Sabot (eds., 1996).

11 América Latina gasta en educación un porcentaje de su PIB tan alto como otros países en desarrollo con niveles similares de ingreso. Pero el hecho de que en América Latina se haya reducido el ritmo de crecimiento significa que los presupuestos de educación han sido menores en términos absolutos. Además, por efecto de la natalidad más alta de América Latina un mismo porcentaje del Pin se traduce en un menor gasto por niño (Birdsall y Sabot, eds., 1996).
} 
sica subvencionada a un segmento grande de la población en edad escolar implica una carga tributaria relativamente grande para los ricos. Es probable que los hogares de altos ingresos se resistan. Un resultado puede ser el financiamiento deficitario de la educación - y la merma en calidad que se ha descrito. Un segundo resultado puede ser la canalización de las subvenciones públicas a la educación superior, cuyos beneficiarios han de ser con más probabilidad los hijos de los ricos. De hecho, como lo muestra el cuadro 4, en América Latina la proporción del gasto público en educación que se destina a la educación superior ha sido alta -más del $20 \%$ en promedio, comparado con el $15 \%$ del Asia oriental. Venezuela y Corea son dos ejemplos extremos. Mientras a principios del decenio de 1990 Venezuela destinaba el 35\% de su presupuesto público de educación a la educación superior, en Corea sólo el $8 \%$ de su presupuesto llegaba a la educación postsecundaria. El gasto público en educación, como porcentaje del PIB era en realidad más alto en Venezuela $(5.1 \%)$ que en Corea $(4.5 \%)$. Sin embargo, después de restar la porción destinada a la educación superior, el gasto público disponible para educación básica como porcentaje del PIB era mucho más elevado en Corea $(3.6 \%)$ que en Venezuela (1.3\%) (PNUD, 1997).

Al dar prioridad a la expansión de la cantidad de educación y a mejorar la calidad en la base de la pirámide educativa, los gobiernos del Asia oriental han estimulado la demanda de educación superior, descansando en gran medida en el sector privado para satisfacerla. En América Latina, las subvenciones gubernamentales han beneficiado en forma desproporcionada a los hogares de altos ingresos cuyos hijos tienen más probabilidad de llegar a la universidad. Al mismo tiempo, el escaso financiamiento público de la educación secundaria significa que los niños mal preparados provenientes de sectores de bajos ingresos, se ven obligados a asistir a las universidades privadas o a quedar totalmente excluidos de los sistemas educativos de nivel superior. El financiamiento deficitario de la educación ha convertido en letra muerta la garantía de la educación primaria universal para los pobres de América Latina; la educación disponible para ellos ha sido de tan mala calidad que les reporta muy poco beneficio real.
CUADRO 4

Presupuesto destinado a la educación superior, 1990-1994

\begin{tabular}{lc}
\hline & $\begin{array}{c}\text { Porcentaje del presupuesto } \\
\text { total en educación }\end{array}$ \\
\hline Asia oriental & 17 \\
Malasia & 17 \\
Tailandia & 18 \\
Indonesia & 8 \\
República de Corea & 15 \\
Promedio (simple) & \\
América Latina & 17 \\
Argentina & 26 \\
Brasil & 20 \\
Chile & 17 \\
Colombia & 31 \\
Costa Rica & 23 \\
Ecuador & 20 \\
Honduras & 14 \\
México & 11 \\
República Dominicana & 25 \\
Uruguay & 35 \\
Venezuela & \\
Promedio (simple) & 22 \\
\hline
\end{tabular}

Fuente: Programa de las Naciones Unidas para el Desarrollo, Desarrollo humano, Informe 1997, Santafé de Bogotá, Tercer Mundo Editores, 1997.

En resumen, el crecimiento económico relativamente bajo de América Latina, la persistencia de desigualdades de ingreso y las dificultades para reducir la pobreza no se pueden separar de la triste trayectoria regional de acceso limitado y desigual a la educación. En una serie de círculos viciosos, los altos niveles históricos de desigualdad de activos e ingresos han generado un ambiente político y económico que limita gravemente las oportunidades de los pobres. Con escasas posibilidades de educación, a pesar de un gasto razonable de los gobiernos, y careciendo de otros activos productivos, los pobres están condenados al trabajo de baja productividad, al bajo ingreso en los hogares y a un nuevo ciclo de acceso limitado a la educación. Las sociedades de la región se ven afectadas también en su conjunto, porque el progreso educativo y la acumulación de activos se limitan a los que no son pobres, reduciéndose los niveles medios de crecimiento y perpetuándose la desigualdad. 


\section{IV}

\section{Conclusiones y reflexiones}

Este artículo comenzó con una crítica, al menos en términos de orientación de políticas, de los modelos actuales de crecimiento endógeno - que apuestan a la educación pero no logran considerar la importancia de la demanda de educación de los hogares, los efectos de la distribución de la educación y los problemas institucionales de la entrega de la educación, especialmente a los pobres. La discusión destaca la importancia de la demanda de escolaridad de los pobres en América Latina, inhibida por muchas décadas por políticas económicas desfavorables a la mano de obra; y la distribución del capital humano, en que el acceso desigual a la escolaridad de los pobres retarda el proceso de acumulación. También se subraya que el acceso limitado y desigual al capital humano que se registra en América Latina se vincula con el bajo crecimiento económico de América Latina, la persistencia de una alta desigualdad en la distribución de los ingresos y las dificultades para reducir la pobreza. Merecen especial atención los datos que sugieren que la distribución de la educación no ha mejorado o lo ha hecho escasamente en los últimos treinta años.

Para comenzar, se proporcionan antecedentes de que la acumulación baja y desigual de capital humano no sólo ha retardado el crecimiento global de América Latina, sino que ha dificultado la reducción de la pobreza y ha contribuido a la persistencia de los más altos niveles mundiales de desigualdad de ingresos. Las consecuencias son claras - hay un gran potencial de crecimiento acelerado para la región. El proceso de crecimiento podría beneficiarse de aumentos importantes de productividad entre los pobres, si se les diera acceso a la educación. Más educación, y en particular, una educación más igualitaria (que llegue a los pobres), podría acelerar el proceso de crecimiento y simultáneamente reducir la desigualdad.

A continuación se sostiene que en América Latina la baja acumulación de capital humano y su distribución desigual tienen su origen, en parte, en una desigualdad, de larga data, de los activos (incluso el propio capital humano) y de los ingresos. El legado histórico es difícil de modificar a corto plazo, pero la acumulación baja y desigual puede también tener su explicación en las políticas económicas que prevalecieron en la región por décadas. Las economías cerra- das que protegían al capital y dependían de la exportación de recursos naturales desalentaban la demanda de educación en los hogares pobres de América Latina, al desalentar la demanda de mano de obra, el activo más importante de los pobres, y de mano de obra calificada, donde radica el mayor potencial de aumento de sus ingresos.

De hecho, la desigualdad de acceso se ha imbuido de un criterio de entrega de servicios sociales que alienta la exclusión, la segmentación y la ineficiencia. Los sistemas burocráticos verticales tradicionales para organizar la enseñanza y otros servicios han sido ineficientes y no han logrado atender a los pobres. El modelo alternativo de entrega privada, orientado por el mercado, no lo ha hecho mejor: en algunos países, la competencia que genera ha dejado fuera del sistema a los que no pueden pagar.

No obstante, hay motivos de optimismo. En la mayoría de los países de la región, las reformas económicas de la última década están eliminando los sesgos contra la mano de obra, típicos de las economías protegidas, y están produciendo la estabilidad macroeconómica esencial para la inversión privada. Se están sentando así las bases para un aumento de la demanda de escolaridad por parte de los hogares y de un mayor interés del sector empresarial en la mano de obra calificada para mantener la competitividad en las economías abiertas. Estas fuerzas se han conjugado para impulsar la reforma educativa en la región y para despertar un renovado interés entre los responsables de la política en nuevos sistemas de provisión de educación, salud y otros servicios públicos. Ya se ha comenzado en algunos países a aplicar sistemas alternativos de provisión de servicios sociales que promueven la competencia entre oferentes públicos y privados, hacen valer el poder del consumidor y su capacidad de opción y aseguran la incorporación integral de los pobres como consumidores al sistema, mediante reglas de acceso justas, sistemas de vales y otros subsidios a la demanda. Estas reformas están demostrando que es posible conciliar la eficiencia con la equidad, ${ }^{12}$

\footnotetext{
12 Sobre enfoques alternativos de provisión de servicios sociales,
} véase Birdsall y Londoño, 1998. 
Los trade offs no son inevitables en el proceso del desarrollo; muchos son resultado de malas decisiones políticas y de instituciones débiles. Centrarse en el acceso igualitario para los pobres a los servicios que generan capital humano, en otras palabras, superar el proceso de filtración puede traer consigo más crecimiento y un mejoramiento de la equidad para las economías y los pueblos de América Latina.

(Traducido del inglés)

\section{Bibliografía}

Alesina, A. y D. Rodrik (1994): Distributive politics and economic growth: A critical survey of the recent literature, The Quarterly Journal of Economías, 109: 465-90, mayo.

Banco Mundial (varios años): Informe sobre desarrollo mundial, Washington, D.C,

Barro, RJ. (1991): Economic growth in a cross section of countries, The Quarterly Journal of Economics, 106: 407-43, mayo.

Barro, R.J. y J. Lee (1993): International Comparisons of Educational Attainment, Washington, D.C, Banco Mundial.

Barro, R,J. y X. Sala-i-Martin (1995); Economic Growth, Nueva York, McGraw Hill.

BID (Banco Interamericano de Desarrollo) (1996): Progreso económico y social en América Latina, Washington D.C.

Birdsall, N. (1998): Education: The people's asset, trabajo preparado para la conferencia Asset Distribution, Poverty and Economic Growth, Brasilia (http://www.worldbank.org/landpolicy/ brazil/papers.htm.

Birdsall, N., B. Bruns y R. Sabot (1996): Educalion in Brazil: Playing a bad hand badly, en N. Birdsall y R. Sabot (eds.), Opportunity Foregone: Education in Brazil, Washington D.C, The Johns Hopkins University Press.

Birdsall N. y J. L. Londoño (1997): Asset inequality matters: An assesment of the World Bank's approach to poverty reduction, The American Economic Review, vol. 87, N² , Nashville, Tennessee, American Economic Association.

(1998): No trade off. Efficient growth via more equal human capital accumulation in Latín America, en N. Birdsall, C. Graham y R. Sabot (eds.), Beyond Trade-Offs: Market Reforms and Equitable Growth in Latin America, Washington, D.C, The Brookings Institution/BID.

Birdsall N., D. Ross y R. Sabot (1995): Inequality and growth reconsidered: Lessons from East Asia, The World Bank Economic Review, vol. 9, $\mathrm{N}^{\circ}$ 3, Washington, D.C, Banco Mundial.

(1997): Education growth and inequality, en N. Birdsall y

F. Jaspersen (eds.), Pathways to Growth: Latin America and East Asia, Washington, D.C, BID.

Birdsall N. y R. Sabot (eds.) (1996): Opportunity Forgone: Education in Brazil, Washington, D.C, The Johns Hopkins University Press.

Deininger, K. y L. Squire (1996): A new data set measuring income inequality, The World Bank Economic Review, vol. 10, $\mathrm{N}^{\circ} 3$, Washington, D.C, Banco Mundial.

Engerman, S.L. y K.L. Sokoloff (1997): Factor endowments, institutions and differential paths of growth among New World economies: A view froni economic historians of the United States, en S. Haber (ed.), How Latín America Fell Behind, Essays on the Economic History of Brazil and México, ;8001914, Stanford, California, Stanford University Press.

Flug, K, A. Spilimbergo y E. Wachtenheim (1996); Investment in Education: Do Economic Volalility and Credit Constraints
Matter? Office of the Chief Economist working paper series, $\mathrm{N}^{\circ} 301$, Washington, D.C, BID.

Levine, R. y D. Renelt (1992): A sensitivity analysis of cross-country growth regressions, The American Economic Review, vol. 82, $\mathrm{N}^{\circ}$ 2, Nashville, Tennessee, American Economic Association.

Londoño, J.L. y M. Székely, (1997): Distributional surprises after a decade of reforms: Latin America in the nineties, R. Hausman y E. Lora (eds.), Latin America after a Decade of Reforms: What Comes Next? Washington, D.C, BID.

Lora, E. y F. Barrera (1997): A decade of structural reforms in Latin America: Growth, productivity and investments are not what they used to be, en R. Hausman y E. Lora (eds.), Latin America after a Decade of Reforms: What Comes Next?, Washington, D.C, BID,

Persson, T. y G. Tabellini (1994): Is inequality harmful for growth? The American Economic Review vol. 84, N 3, Nashville, Tennessee, American Economic Association.

PNUD (Programa de las Naciones Unidas para el Desarrollo) (1997): Desarrollo humano. Informe 1997, Santafé de Bogotá, Tercer Mundo Editores.

Schiff.M. y A. Valdés(1992): The Politkal Bamamy of Agricultural Pricing Policy. Volume 4: A Synthesis of the Economics in Developing Countries, Baltimore, Pennsylvania, Johns Hopkins University Press.

Schiefelbein, E. (1995): Characteristics of the Teaching Profession and the Quality of Education in Latin America. Bulletín: The major project of education in Latin America and the Caribbean, $\mathrm{N}^{\circ} 34$, Santiago de Chile, Organización de las Naciones Unidas para la Educación, la Ciencia y la Cultura (UNESCO).

Schultz, T.P. (1988): Education investment and returns, en H. B. Chenery y T,N. Srinivasan (eds.): Handbook of Development Economics, Amsterdam, Países Bajos, North-Holland Publishing Company.

Stallings B., N. Birdsall y J. Clugage (por publicarse): Growth and inequality: Do regional patterns redeem Kuznets?, en A. Solitnano (ed.) Distributive Justice and Economic Development: The Experience of Chile and Developing Countries, Ann Arbor, Michigan, University of Michigan Press.

Summers, R. y A. Heston (1995): The Penn World Tables, Mark 5.6. Philadelphia Pa., University of Pennsytvania, Department of Economics.

UNESCO (Organización de las Naciones Unidas para la Educación, la Ciencia y la Cultura) (varios años): Statistical Yearbook, Nueva York, Bernan Press.

Williamson, J. (1993): Human capital deepening, inequality, and demographic events along the Asia-Pacific rim, en N. Ogawa, G.W. Jones y J. Williamson (eds,), Human Resources in Development along the Asia-Pacific Rim, Singapore, Oxford University Press. 